\title{
Non-destructive controlled single-particle light scattering
}

measurement

G. Maconi ${ }^{1,}{ }^{*}$, A. Penttilä ${ }^{1}$, I. Kassamakov ${ }^{1}$, M. Gritsevich ${ }^{1,2}$, P. Helander ${ }^{1}$, T. Puranen ${ }^{1}$, A. Salmi ${ }^{1}$, E.

5 Hæggström ${ }^{1}$, and K. Muinonen ${ }^{1,3}$

${ }^{1}$ Department of Physics, University of Helsinki, Finland

${ }^{2}$ Institute of Physics and Technology, Ural Federal University, Ekaterinburg, Russia

${ }^{3}$ Finnish Geospatial Research Institute FGI, National Land Survey of Finland

"corresponding author. E-mail: goran.maconi@helsinki.fi

\section{Abstract}

We present a set of light scattering data measured from a mm-sized extraterrestrial rock sample. The data were acquired by our novel scatterometer, which enables accurate multi-wavelength measurements of single-particle samples whose position and orientation are controlled by ultrasonic levitation. The measurements demonstrate a non-destructive approach to derive optical properties of small mineral samples. This enables research on valuable materials, such as those returned from space missions or rare meteorites.

Keywords: light scattering, optical properties, ultrasonic levitation, space mission

\section{Introduction}

Non-destructive measurements are important for characterizing unique and valuable samples, e.g. samples returned from Solar System objects (samples from the asteroid Itokawa and from the Moon). Scattering theory provides tools for characterizing small particles. By examining the light scattered by a particle, one can estimate its optical properties or its shape. Since the inverse problem does not necessary have a unique solution, one may need assumptions on, e.g., constant bulk optical properties throughout the particle's volume, shape model for the particle, or a measured shape estimate using, for example, microscopy, laser scanning, X-ray microtomography, or even holography[1]. Scattering-based imaging could create a pathway towards super-resolution imaging [2]. In the scattering field, there are difficulties in combining modeled and measured results for small particles or particle aggregates. Exact methods exist for modeling the scattering response of complex small particles, but these methods are computationally demanding and thus limited with respect to size of the particle, compared to the wavelength [3]. On the other hand, scattering measurements, together with target structure characterization, are more conveniently done for larger particles or aggregates. For small particles, it is easier to measure the average scattering characteristics for a particle population, e.g., particles in a flow [10] or on a particulate surface $[4,5,6,7]$, than to measure individual small-particle scattering for identified particles. One of the first scatterometry setups for small particle characterization was built in Arizona [8]. The system was used to characterize $110 \mathrm{~nm}$ diameter latex spheres. The light source was a high-pressure $\mathrm{Hg}$ lamp (Osram HBO 100). To acquire all the elements of the Mueller matrix, polarized light was used. Later, another system was built in the Netherlands, to measure scattering properties of irregularly shaped mineral aerosol samples [9]. This system was further developed at the IAA cosmic dust laboratory, Granada, Spain[10] to become one of the first systems to truly bridge the gap between theory and 
experiment[11], albeit with the limitation of measuring the statistical average of a group of particles. A comparison across these three setups, as well as our own, is shown in Appendix 1. Lately, two more systems have been developed [12,13]. The latest one allows characterization of levitated water droplets using a $100 \mathrm{kHz}$ ultrasound levitator. As a light source, it uses a collimated, vertically polarized, coherent and continuous laser beam, with wavelength $\lambda=532.130 \mathrm{~nm}$ (coherence length $50 \mathrm{~m}$ and high laser power $2 \mathrm{~W}$ ).

We present an experimental setup that measures light scattered by a fixed particle in the size range of micrometers to millimeters. The goal of our setup is nondestructive characterization of such particles by measuring light of multiple wavelengths scattered in $360^{\circ}$ in a horizontal plane by a levitating sample, whilst simultaneously controlling its 3D position. This work describes the principles and design of our instrument and its calibration. It also presents an example measurement of a real sample, as well as discussing some of the considerations that have to be made when choosing the light source.

\section{Scattering theory}

We describe the polarization state of light beam using the Stokes parameters $I, Q, U$, and $V$, which we combine into a vector known as the Stokes vector. A scattered beam can thus be related to an incoming beam by multiplication with the $4 \times 4$ scattering matrix, $S$. For an incoming vector $I_{i}=\left(I_{i}, Q_{i}\right.$, $\left.U_{i}, V_{i}\right)^{T}$ and a scattered vector $I_{s}=\left(I_{s}, Q_{s}, U_{s}, V_{s}\right)^{T}$ the transformation becomes:

$$
\boldsymbol{I}_{S}=\frac{1}{k^{2} R^{2}} \boldsymbol{S} \boldsymbol{I}_{\boldsymbol{i}}
$$

Here $R$ is the distance from the particle to the observer and $k$ is the wave number. Individual elements of the scattering matrix can be defined by measuring the scattered light at specific polarization states. To resolve all the matrix elements, the polarization of the incoming light is controlled by a linear polarizer and a quarter wave plate [14]. Since our detector measures only light intensity, the scattered light must also be filtered by an equivalent polarizer and a quarter wave plate. The Mueller matrices for a linear polarizer $\mathbf{M}_{p}$ and a quarter wave plate $\mathbf{M}_{q}$ are:

$$
\begin{gathered}
\mathbf{M}_{p}(\theta)=\frac{1}{2}\left(\begin{array}{cccc}
1 & \cos (2 \theta) & \sin (2 \theta) & 0 \\
\cos (2 \theta) & \cos ^{2}(2 \theta) & \sin (2 \theta) \cos (2 \theta) & 0 \\
\sin (2 \theta) & \sin (2 \theta) \cos (2 \theta) & \sin ^{2}(2 \theta) & 0 \\
0 & 0 & 0 & 0
\end{array}\right) \\
\mathbf{M}_{q}(\theta)=\frac{1}{2}\left(\begin{array}{cccc}
1 & 0 & 0 & 0 \\
0 & \cos ^{2}(2 \theta) & \sin (2 \theta) \cos (2 \theta) & \sin (2 \theta) \\
0 & \sin (2 \theta) \cos (2 \theta) & \sin ^{2}(2 \theta) & -\cos (2 \theta) \\
0 & -\sin (2 \theta) & \cos (2 \theta) & 0
\end{array}\right)
\end{gathered}
$$

Thus, for one single measurement with the scatterometer, the chained transformation becomes:

$$
\boldsymbol{I}_{s}=\mathbf{M}_{p}\left(\theta_{4}\right) \mathbf{M}_{q}\left(\theta_{3}\right) \mathbf{M} \mathbf{M}_{q}\left(\theta_{2}\right) \mathbf{M}_{p}\left(\theta_{1}\right) \boldsymbol{I}_{i}
$$

where $\mathbf{M}=k^{-2} R^{-2} \mathbf{S}$. While both the quarter wave plates and the polarizers are needed to determine all 16 elements of the scattering matrix, the upper left $2 \times 2$ submatrix can be determined using only linear polarizers, with, e.g., the following configurations: 


$$
\begin{gathered}
I_{1}: \theta_{1}=0^{\circ} ; \theta_{4}=0^{\circ} \\
I_{2}: \theta_{1}=90^{\circ} ; \theta_{4}=90^{\circ} \\
I_{3}: \theta_{1}=0^{\circ} ; \theta_{4}=90^{\circ} \\
I_{4}: \theta_{1}=90^{\circ} ; \theta_{4}=0^{\circ}
\end{gathered}
$$

From these measurements, the Mueller matrix elements can be calculated as:

$$
\begin{aligned}
& M_{11}=I_{1}+I_{2}+I_{3}+I_{4} \\
& M_{12}=I_{1}-I_{2}+I_{3}-I_{4} \\
& M_{21}=I_{1}-I_{2}-I_{3}+I_{4} \\
& M_{22}=I_{1}+I_{2}-I_{3}-I_{4}
\end{aligned}
$$

\section{The scatterometer}

We developed an automated scatterometer that measures scattered light at different wavelengths from small particulate samples. The setup comprises: (a) the PXI Express platform (National Instruments) to synchronously record data from several photomultiplier tubes (PMTs); (b) a motorized rotation stage to precisely control the azimuthal angle of the PMTs around $360^{\circ}$; (c) a custom-built ultrasonic levitator to hold the sample in a non-contacting way; and (d) a versatile light source, whose wavelength, polarization, and intensity can be precisely controlled.

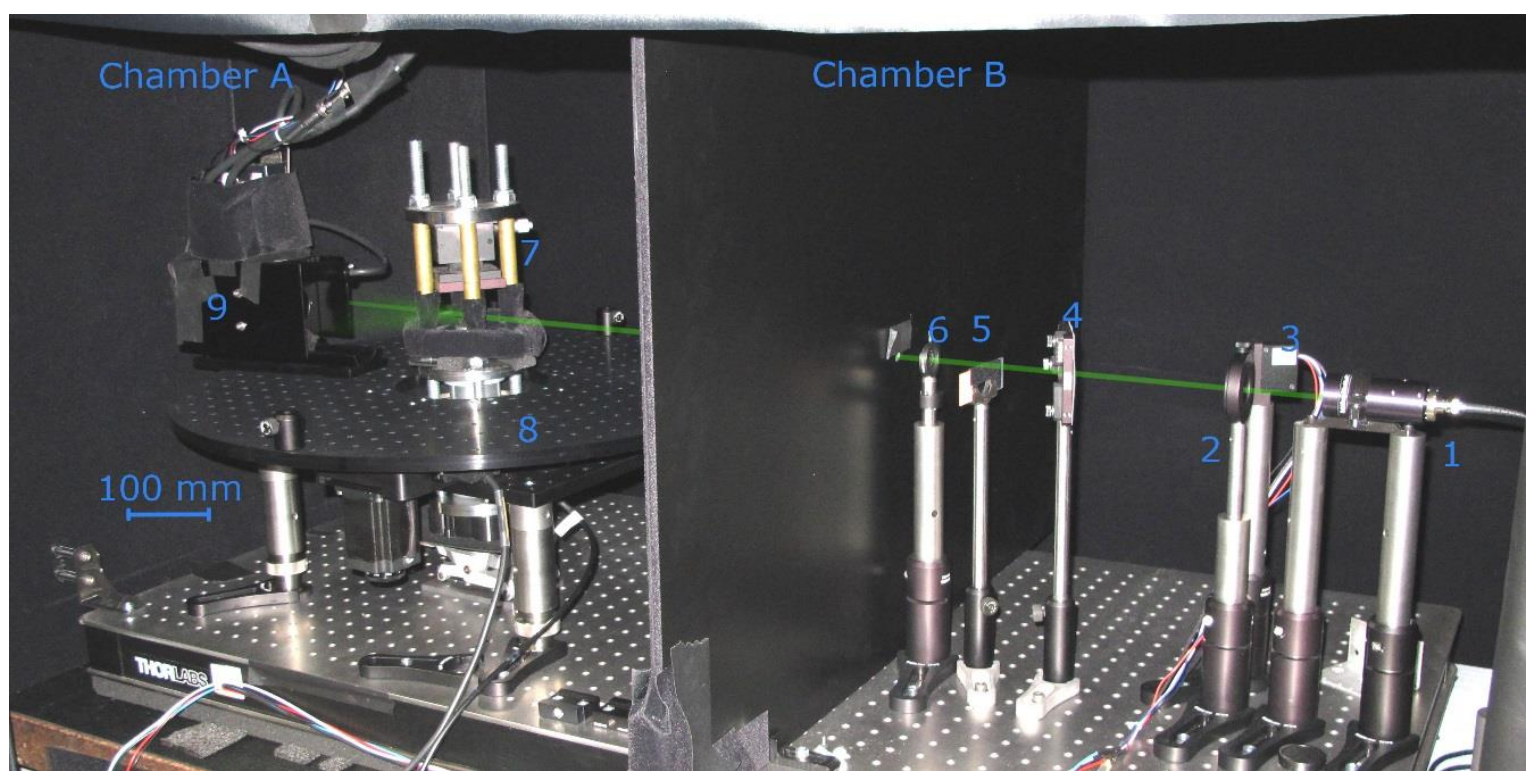

Figure 1: Overview of the scatterometer, comprising 1) fiber and collimator, 2) linear polarizer, 3) reference PMT, 4) diffuse reflector with central aperture, 5) blocking plate for beam shaping, 6)

circular aperture, 7) sample levitator, 8) rotating baseplate, and 9) measurement head, with shutter, linear polarizer, and PMT. A green line is drawn to indicate the beam path.

The light source is a tunable Argon-krypton laser (Melles Griot 35 KAP 431), with 12 wavelengths ranging from 465 to $676 \mathrm{~nm}$, beam quality factor $M^{2}=1.2$, and a power of 4 to $20 \mathrm{~mW}$, depending on wavelength. The polarization of light is selected by linear polarizers (Thorlabs LPVISE100-A). As light detectors, we use photomultiplier tubes (PMT) manufactured by Hamamatsu (microPMT H12403-01, peak sensitivity wavelength $420 \mathrm{~nm}$ ) mounted radially from the sample at adjustable radii, as well as one reference PMT monitoring beam intensity. The PMT signals are amplified by Thorlabs TIA60 transimpedance amplifiers and captured by an NI PXIe-5171R oscilloscope module. 
The current setup allows measuring all azimuthal angles except for $\pm 11^{\circ}$ around the backward and forward scattering directions. These "dead zones" are due to the measurement head blocking the incoming or outgoing beam. For specific applications, this can be improved by replacing the motorized rotation of the polarizer and the motorized shutter in front of the PMT with manual controls, resulting in a narrower $\left( \pm 4^{\circ}\right)$ measurement head. A rotation stage (Standa 8MRB240-15259D) controlled through the NI PXIe-8880 moves the PMTs. The measurement angle is controlled by the motor-driven rotation stage with an accuracy of $15^{\prime}$.

To reduce external light and reflections, the device is enclosed inside three adjacent chambers covered with a black velvet-like material. The first chamber contains beam shaping and filtering optics, the second contains the sample and sensors, whereas the third chamber contains the beam stop. The laser is outside the enclosure for improved temperature control. The light is transferred from the laser by a polarization-maintaining fiber (single mode, pure silica, PANDA geometry) into the first chamber, where the light is collimated.

The sample is held in place in a noncontacting manner by a custom-built ultrasonic levitator, Fig. 2 . The standing ultrasonic waves are tuned to create a node at a suitable height in the acoustic field, trapping the sample. The levitator is based on a Langevin transducer, with two sandwiched piezoelectric discs (Ferroperm Pz26, $\varnothing=50 \mathrm{~mm}$, thickness $=1 \mathrm{~mm}$ ), driven at a frequency of $35.2 \mathrm{kHz}$ with an electrical power of $2.5 \mathrm{~W}$. To ensure that the sample is centered in the device, the levitator position can be adjusted in the $x$ - $y$ - and $z$-directions. For large samples, e.g. the 5-mm glass sphere used for calibration, the levitator can be replaced by a static conic pedestal.

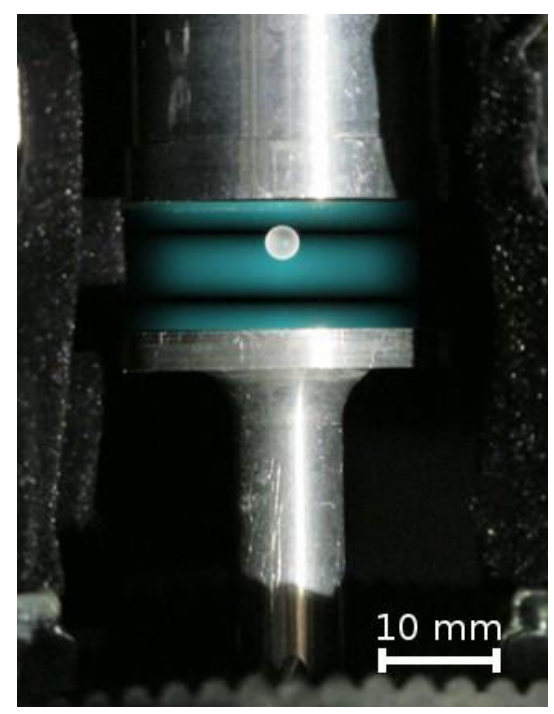

Figure 2: Closeup of the acoustic sample levitator, holding a 3-mm frosted glass sphere. A visualization of the acoustic field is drawn in blue.

The Langevin-type levitator does not control the rotation $(\rho, \theta, \phi)$ of the sample, but it does provide a well-defined potential minimum which keeps the sample in position $(x, y, z)$. Using a high-speed camera (Phantom V611) we measured the radial and axial movement of millimeter-sized rock samples. Automated tracking of the lit area of the sample revealed that, for the worst-case oscillations, average displacement was $700 \mu \mathrm{m}$ in the radial direction and $70 \mu \mathrm{m}$ in the axial direction, Fig. 3. 


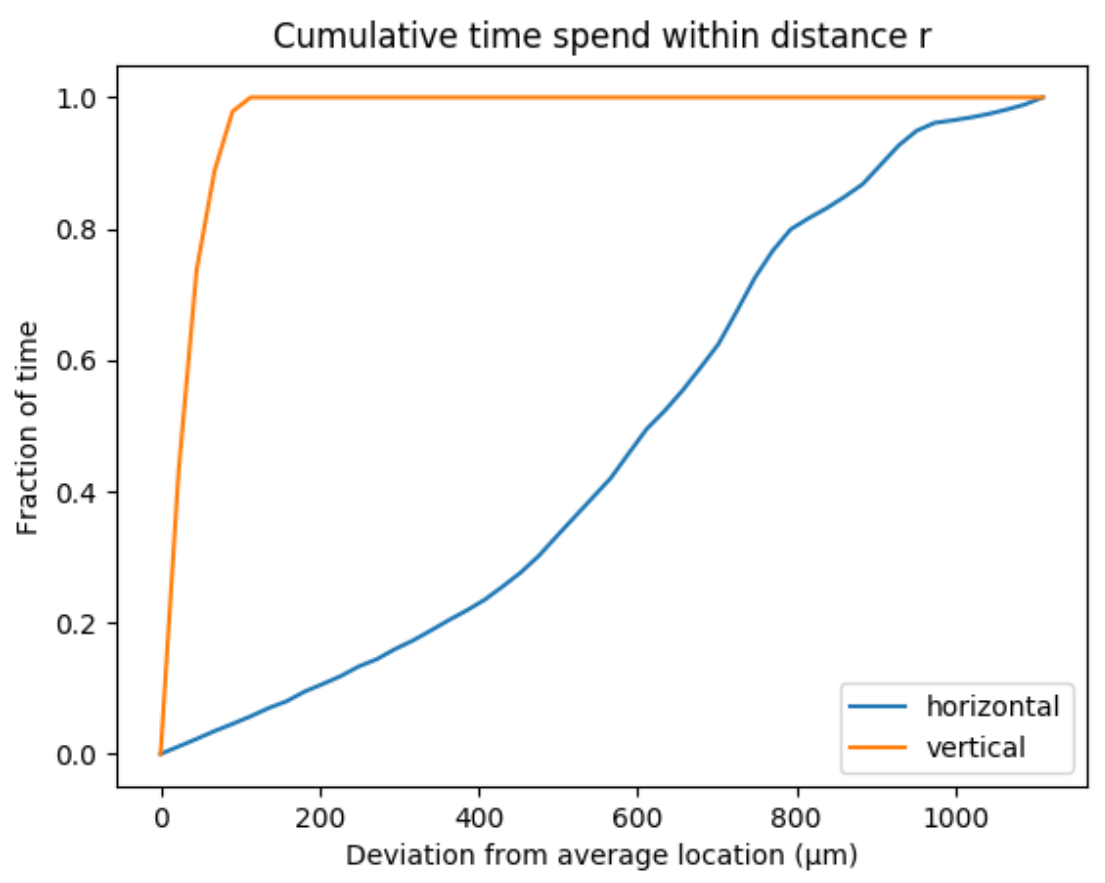

Figure 3: Worst-case displacement characteristics of a $1 \mathrm{~mm}$ rock sample

The PXIe-8880 module acquires timing-critical data from PMTs and motor drivers. The signals from the PMTs transimpedance amplifier are averaged over the exposure time for each angle, to reduce the amount of stored data. The system is programmed with LabVIEW, including FPGA-based signal acquisition, data processing and the instrument's user interface.

135 The scatterometer measures the scattered light by sweeping the PMT across a specified range of angles, measuring at each angular step for a set exposure time. Each sweep is made for only one wavelength and polarizer configuration. A complete measurement consists of one or more sweeps for each of the four polarizer configurations described in the theory section. Changing the wavelength is typically only done between measurements, since it requires a manual and precise adjustment of the laser's reflective grating. For the calibration measurements, we used an angular step of $0.25^{\circ}$ and an exposure time of $300 \mathrm{~ms}$ per step. To avoid artifacts from temporary disturbances, we measured three consecutive sweeps for each polarizer configuration, resulting in a total of 12 sweeps per measurement that takes roughly four hours to complete. The laser output power was $10 \pm 1 \mathrm{~mW}$ for the wavelength used $(514 \mathrm{~nm})$, as measured internally by the laser's optical power meter, as well as externally using a Coherent LabMax laser power meter. The fiber coupling achieved a transmission efficiency of $35 \pm 5 \%$, which reduced the transmitted power to $3 \mathrm{~mW}$. The light was then collimated into a $25 \mathrm{~mm}$ wide beam with a gaussian intensity profile that was clipped so that the light covers the whole sample, without illuminating the levitator or sample holder.

\section{System alignment and calibration}

150 Before performing a measurement, the system needs to be aligned so that both the beam and the detectors are in the same horizontal plane, and the sample is in the center of both the beam and the axis of rotation. The rotation stage is leveled using a digital 2-axis level (Digi-pas DWL3500XY). To level the beam, two pin holes are mounted to the rotation stage at equal height. The collimator is then adjusted in such a way that the laser beam passes through both pin holes. For sample 
positioning, the laser beam is crossed with itself at a right angle, using two mirrors, Fig. $4 a$, b. The crossing point of the beam indicates the precise point of measurement in three dimensions. The rotating stage where the mirrors and pinholes for the calibration are attached is a breadboard with accurately-positioned screw holes. The correct angles are found by rotating the mirrors so that the beam path in fig. 4 is achieved. Once done, the crossing point of the beams is in the middle of the rotating stage. By manual inspection using this method, one can achieve an uncertainty in position of $\pm 0.2 \mathrm{~mm}$, which, at a radius of $300 \mathrm{~mm}$, gives an angular uncertainty of $\pm 2.3^{\prime}$, i.e. better than the accuracy of the rotational stage.

a)
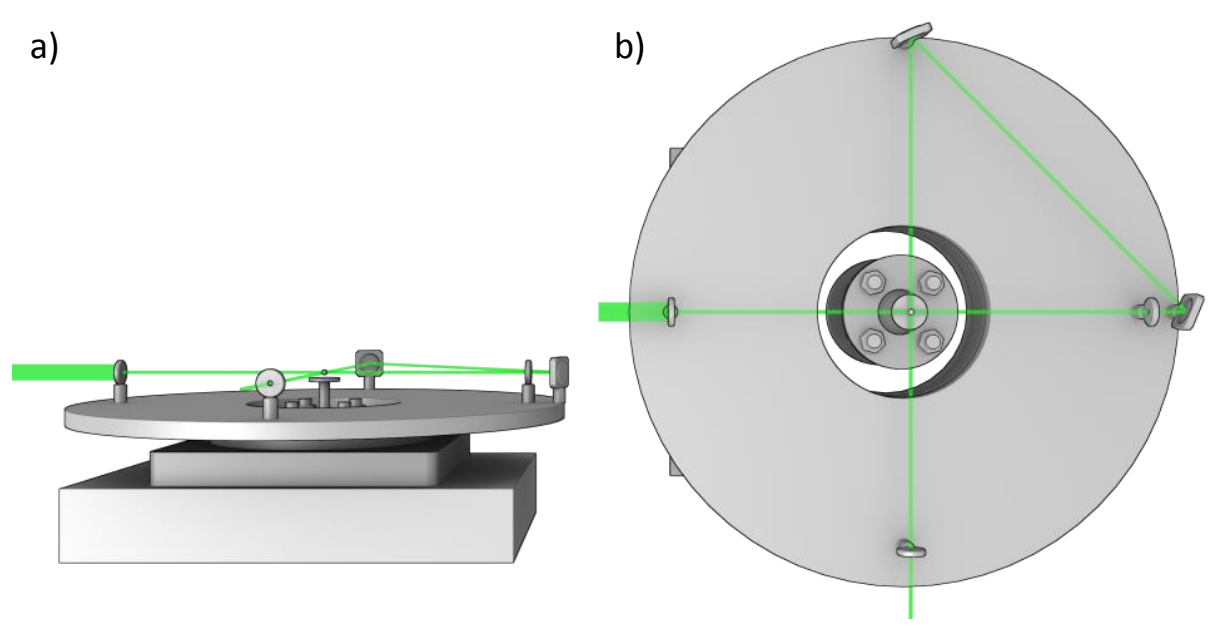

Figure 4: Schematic of the beam path during alignment. a) Side view, b) Top view.

The system was calibrated by measuring a clear glass sphere ( $d=5 \mathrm{~mm} \pm 5 \mu \mathrm{m}, \mathrm{N}$-BK7 glass, $\mathrm{n}=$ 1.517, Edmund optics), using a 514-nm laser beam [15]. We measure four different polarization configurations to obtain four elements of the Mueller matrix. Figure 5 shows results for the verticalvertical and horizontal-horizontal configurations of the polarizers. Furthermore, we modelled the scattering properties of clear glass spheres with the abovementioned parameters using the Mie theory, and averaging the results over an angular window of 2.25 degrees.
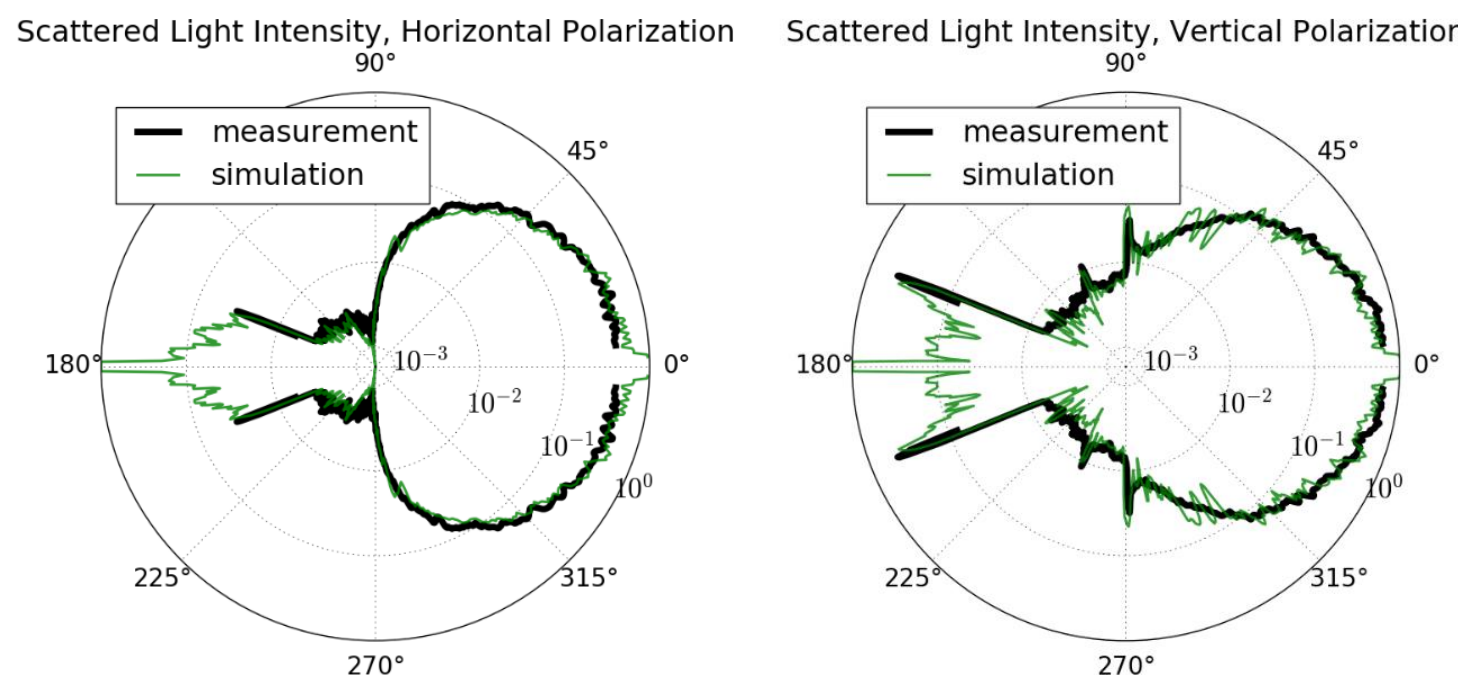
Figure 5: Comparison between simulated and measured light scattering from a 5-mm glass sphere, as a function of scattering angle. The measurement data is scaled to fit the normalized simulation data.

\section{Measurement results}

To demonstrate the capacity of our instrument, and its suitability for real-world applications, we measured a number of mineral samples including a mm-sized rock sample taken from the lightcolored lithology sample of the Chelyabinsk meteorite [16], Fig. 6. The opaque rock sample has a high amount of backscattering which gradually drops off, but with a peak around the forward scattering direction.

a)

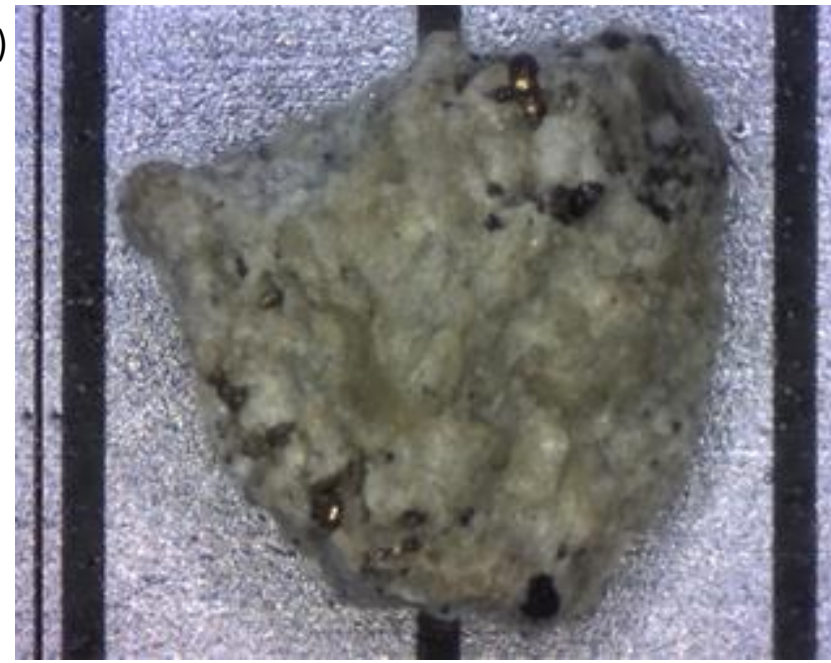

b)

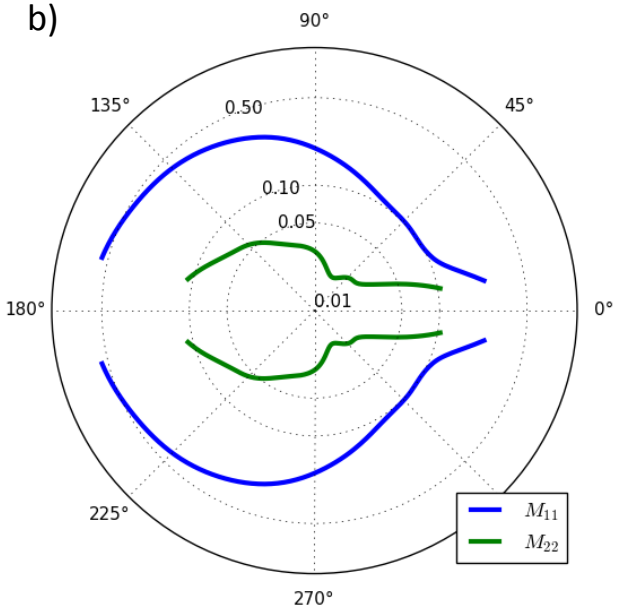

185

Figure 6: a) the measured sample, a Chelyabinsk LL5 chondrite particle, derived from the lightcolored lithology sample of the meteorite. Black lines in the background indicate millimeter steps. b) Scattering matrix elements $M_{11}$ and $M_{22}$ as a function of scattering angle (normalized intensities).

The high backscattering is expected from a macroscopic sample like this one, since the thickness and internal structure of the particle heavily reflects and diffuses the incoming light. This also results in a relative lack of features in the $\mathrm{M}_{11}$ curve, compared to e.g. the clear glass sphere, since the surface roughness breaks up any such patterns. For simulation, the sample could be treated as an agglomeration of small particles with multiple scattering. With the positional control, the described approach gives us the capability to obtain a set of data from a meteorite sample, which is more comprehensive than conventionally used techniques [17].

\section{Discussion}

The Argon-Krypton gas laser provides a stable, coherent, and monochromatic light source. However, the long coherence length sometimes gives rise to unwanted optical phenomena, like interference fringes. This problem was pronounced when using birefringent polarizers, which otherwise have favorable properties. Our adjustable waveplates (Newport 5540) also displayed interference fringes, resulting in an inhomogeneous beam intensity. As a solution, the birefringent polarizers were replaced with dichroic sheet polarizers. The waveplates were omitted entirely for the time being, limiting our measurement capabilities to only 4 of the Mueller matrix elements. To ensure that our 
sheet polarizers were suitable for the instrument, we investigated their influence on the measurement results. For this purpose, we measured our calibration sample in vertically polarized light, 1) without polarizers (the polarization is produced by the laser itself), 2) with polarizers in place, facing the beam at a right angle, and 3) with the input polarizer tilted 20 degrees. Every configuration was measured three times and the results are presented in Fig. 7. The polarizers in the system produced no artifacts that could be detected in repeated measurements.

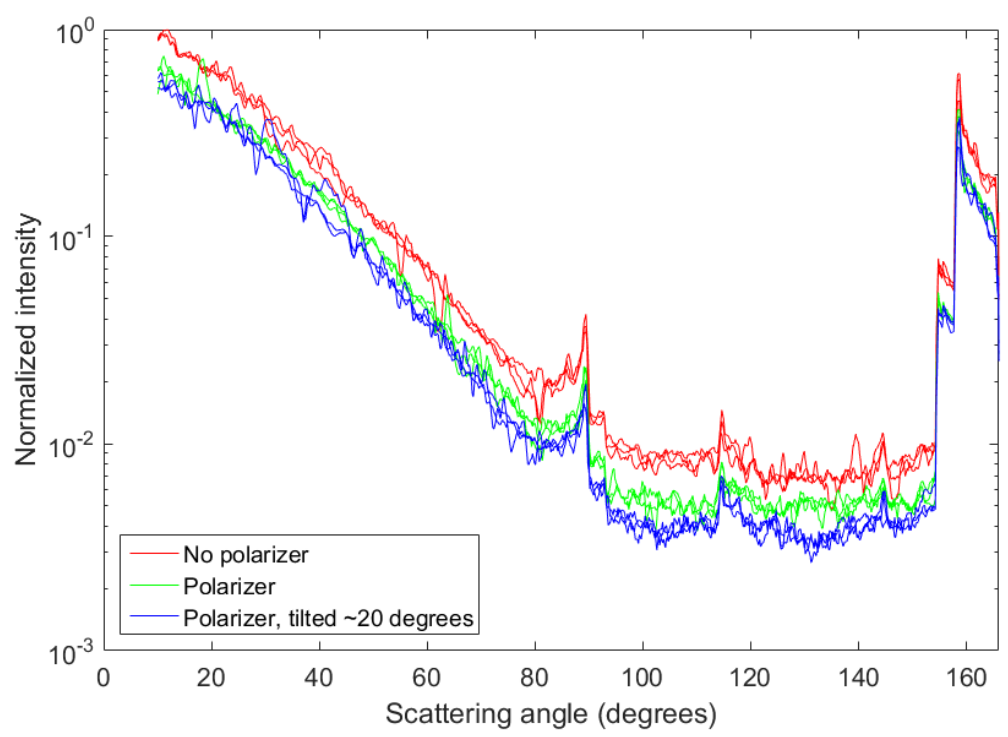

Figure 7: Effect of polarizers on the measured light scattering intensity. Only a minor uniform drop in light intensity can be detected.

Since the laser is not focused on the sample, the absorbed light intensity is low ( $<0.05 \mathrm{~mW} / \mathrm{mm} 2)$. The effect of the light on our mineral samples is therefore negligible. However, the long exposure time can make our setup less suited for sensitive organic and/or volatile samples. While not an issue in our current research, this energy issue can be alleviated by adding multiple PMT:s to the rotating breadboard.

\section{Conclusions}

Our scatterometer is a valuable tool for measuring optical properties of levitated small particles. The tool can be used to validate theoretical models. It provides unique measurement data to gain new knowledge about the optical properties of both individual, and groups of small particles, including planetary materials. The device is the first of its kind, since it measures spectral angular scattering, at different polarizations, for an arbitrary object in the $\mu \mathrm{m}$ - $\mathrm{mm}$ size scale. It permits a non-destructive, disturbance-free measurement with control of the orientation and location of the scattering object.

\section{Acknowledgements}

We acknowledge the ERC Advanced Grant no. 320773 entitled "Scattering and Absorption of Electromagnetic Waves in Particulate Media" (SAEMPL).

We thank Olga Muñoz as well as the rest of the team at the IAA cosmic dust laboratory for providing invaluable information and advice regarding the design of our instrument. 
We are grateful to the editor, Matthew Berg, and the anonymous reviewer for their valuable feedback which helped us improve the original version of this manuscript.

235 The research at the UrFU are supported by the Government of the Russian Federation (Act 211, agreement 02.A03.21.0006).

\section{References}

[1] Berg, M.J. and Videen, G., Digital holographic imaging of aerosol particles in flight. JQSRT 112(11), 1776-1783 (2011).

[2] Ding, H. X., Li, K. Y., Deka, G., Su, I. C., \& Chu, S. W., Resolution enhancement down to 10-nm based on saturated excitation (SAX) microscopy plus novel nonlinear response (Conference Presentation). In SPIE BiOS (pp. 100710G-100710G). International Society for Optics and Photonics (2017).

245 [3] Mishchenko, M.I., Travis, L.D., Lacis, A.A., Scattering, absorption and emission of light by small particles. Cambridge University Press (2002).

[4] Videen, G., Kocifaj, M. (Eds.), Optics of cosmic dust (NATO Science Series). Kluwer Academic Publishers (2002).

[5] Peltoniemi, J.I., Gritsevich, M., Hakala, T., Dagsson-Waldhauserová, P., et al., Soot on snow experiment: bidirectional reflectance factor measurements of contaminated snow. The Cryosphere, 9, 2323-2337 (2015).

[6] Zubko N., Gritsevich M., Zubko E., Hakala T., Peltoniemi J.I., Optical measurements of chemically heterogeneous particulate surfaces. JQSRT 178, 422-431 (2016).

[7] Wilkman, O., Gritsevich, M., Zubko, N., Peltoniemi, J., Muinonen, K., Photometric modelling for laboratory measurements of dark volcanic sand. JQSRT 185, 37-47 (2016).

[8] Hunt, A. J., \& Huffman, D. R., A new polarization-modulated light scattering instrument. Review of Scientific Instruments, 44(12), 1753-1762 (1973).

[9] Rol, E., Vassen, J. D. H. W., \& Hovenier, J. W., Scattering matrices of mineral aerosol particles. Journal of Geophysical Research, 106(D15), 17-375 (2001).

260 [10] Muñoz, O., Moreno, F., Guirado, D., Ramos, J. L., López, A., Girela, F., Jerónimo, J. M., Costillo, L. P., \& Bustamante, I., Experimental determination of scattering matrices of dust particles at visible wavelengths: The IAA light scattering apparatus. JQSRT 111(1), 187-196 (2010).

[11] Muñoz, O., Moreno, F., Guirado, D., Dabrowska, D. D., Volten, H., Hovenier, J. W., The Amsterdam-Granada light scattering database. JQSRT 113(7), 565-574 (2012).

265 [12] Onofri, F. R., Krzysiek, M. A., Barbosa, S., Messager, V., Ren, K. F., \& Mroczka, J., Near-criticalangle scattering for the characterization of clouds of bubbles: particular effects. Applied Optics, 50(30), 5759-5769 (2011).

[13] Onofri, F. R., Ren, K. F., Sentis, M., Gaubert, Q., \& Pelcé, C., Experimental validation of the vectorial complex ray model on the inter-caustics scattering of oblate droplets. Optics Express, 23(12), 15768-15773 (2015). 
[14] Penttilä, A., Maconi, G., Kassamakov, I., Gritsevich, M., Helander, P., Puranen, T., Hæggström, E.,Muinonen, K., Experimental light scattering by small particles: First results with a novel Mueller matrix scatterometer. Proc. SPIE 10330, Modeling Aspects in Optical Metrology VI, 103300B (2017).

[15] Maconi, G., Kassamakov, I., Penttilä, A., Gritsevich, M., Hæggström, E., \& Muinonen, K., 275 Experimental light scattering by small particles: system design and calibration. Optical Measurement Systems for Industrial Inspection X (Vol. 10329, p. 103292S). International Society for Optics and Photonics (2017).

[16] Kohout, T., Gritsevich, M., Grokhovsky, V., Yakovlev, G., Haloda, J., Halodova, P., Michallik, R., Penttilä, A., Muinonen, K., Mineralogy, reflectance spectra, and physical properties of the

280 Chelyabinsk LL5 chondrite - Insight into shock-induced changes in asteroid regoliths. Icarus, 228, 7885 (2014).

[17] Martikainen J., Penttilä A., Gritsevich M., Lindqvist H., Muinonen K., Spectral modeling of meteorites at UV-Vis-NIR wavelengths. JQSRT, submitted 


\begin{tabular}{|c|c|c|c|c|}
\hline & Reference [5] & Reference [6] & Reference [7] & This work \\
\hline Reference & $\begin{array}{l}\text { Rev. Sci. Instrum ., Vol. } \\
\text { 44. No. } 12 \text {, p. } 753,1973\end{array}$ & $\begin{array}{l}\text { J Geophys Res., Vol.1 } \\
\text { 06, p. } 17375,2001\end{array}$ & $\begin{array}{l}1 \text { JQS \& RT, Vol. 111, p. } \\
\text { 187, } 2010\end{array}$ & \\
\hline Light source & $\begin{array}{l}\text { High pressure Hg lamp } \\
\text { (Osram HBO 100) or a } \\
\text { laser }\end{array}$ & $\begin{array}{l}\text { Linearly pol., CW, He- } \\
\mathrm{Ne}(\lambda-632.8 \mathrm{~nm}, 5 \\
\mathrm{mW}) \\
\text { or He-Cd }(\lambda-441.6 \\
\mathrm{nm}, 40 \mathrm{~mW})\end{array}$ & $\begin{array}{l}\text { Linearly pol., } \mathrm{CW} \\
\text { tunable } \mathrm{Ar}-\mathrm{Kr} \lambda-483 \text {, } \\
488,520,568 \text {, or } \\
647 \mathrm{~nm})\end{array}$ & $\begin{array}{l}\text { Argon } \\
\text { krypton laser (Melles } \\
\text { Griot 35 KAP 431) }\end{array}$ \\
\hline $\begin{array}{l}\text { Components after } \\
\text { light source }\end{array}$ & $\begin{array}{l}\text { Neutral density filters, } \\
\text { Polarizer, Slit, } \\
\text { AOModulator, Slit }\end{array}$ & $\begin{array}{l}\text { Polarizer, } \\
\text { AOModulator, } \\
\text { Pinholes }\end{array}$ & $\begin{array}{l}\text { Filter wheel, Polarizer, } \\
\text { AOModulator, Pinhole }\end{array}$ & $\begin{array}{l}\text { Polarizer, Reference } \\
\text { detector, Apertures }\end{array}$ \\
\hline Detector & PMT EMI 9781B & & $\begin{array}{l}\text { 9828A Electron } \\
\text { tubes } \AA\end{array}$ & $\begin{array}{l}\text { Hamamatsu } \\
\text { microPMT } \\
\text { H12403-01 }\end{array}$ \\
\hline $\begin{array}{l}\text { Detector's field of } \\
\text { view }\end{array}$ & & $\sim 2^{\circ}$ & & \\
\hline $\begin{array}{l}\text { Detector step } \\
\text { movement }\end{array}$ & & $1^{\circ}$ or $5^{\circ}$ & $\begin{array}{l}1^{\circ} \text { or } 5^{\circ}, 12 \text { bits } \\
\text { encoders }\end{array}$ & $0.18^{\circ}$ (minimum) \\
\hline $\begin{array}{l}\text { Positioning ring - } \\
\text { outer diameter }\end{array}$ & & & $1 \mathrm{~m}$ & $600 \mathrm{~mm}$ \\
\hline $\begin{array}{l}\text { Scattering angle } \\
\text { range }\end{array}$ & $0^{\circ}(?)-165^{\circ}$ & $3^{\circ}-174^{\circ}$ & $3^{\circ}-177^{\circ}$ & $4^{\circ}-175^{\circ}$ \\
\hline
\end{tabular}

